\title{
The SoftHand Pro: Translation from Robotic Hand to Prosthetic Prototype
}

\author{
Sasha B. Godfrey, Matteo Bianchi, Kristin Zhao, Manuel Catalano, \\ Ryan Breighner, Amanda Theuer, Karen Andrews, Giorgio Grioli, \\ Marco Santello and Antonio Bicchi
}

\begin{abstract}
This work presents the translation from a humanoid robotic hand to a prosthetic prototype and its first evaluation in a set of 9 persons with amputation. The Pisa/IIT SoftHand is an underactuated hand built on the neuroscientific principle of motor synergies enabling it to perform natural, human-like movements and mold around grasped objects with minimal control input. These features motivated the development of the SoftHand Pro, a prosthetic version of the SoftHand built to
\end{abstract}

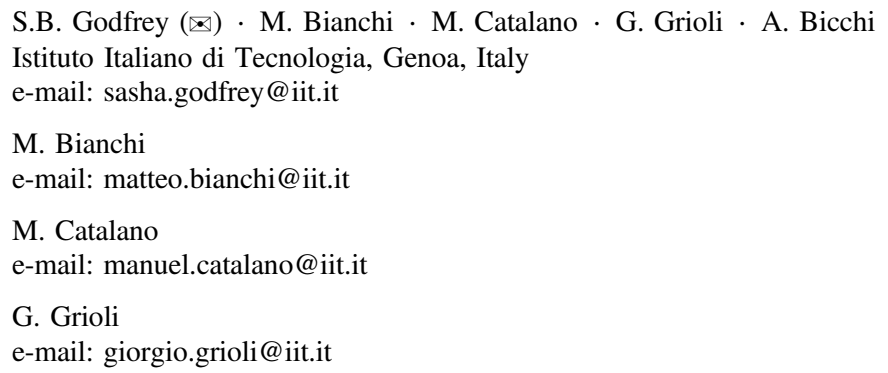
A. Bicchi
e-mail: antonio.bicchi@iit.it
K. Zhao · R. Breighner · A. Theuer · K. Andrews
Mayo Clinic, Rochester, USA
e-mail: zhao.kristin@mayo.edu
R. Breighner
e-mail: breighnerr@hss.edu

\author{
A. Theuer \\ e-mail: theuer.amanda@mayo.edu \\ K. Andrews \\ e-mail: andrews.karen@mayo.edu \\ M. Santello \\ Arizona State University, Tempe, USA \\ e-mail: marco.santello@asu.edu \\ M. Bianchi · M. Catalano · G. Grioli · A. Bicchi \\ University of Pisa, Pisa, Italy
}


interface with a prosthetic socket. The results of the preliminary testing of the SoftHand Pro showed it to be a highly functional design with an intuitive control system. Present results warrant further testing to develop the SoftHand Pro.

\section{Introduction}

Prosthetic function and satisfaction remain low despite advances in technology. Although myoelectric prostheses, those driven by residual muscle signals and powered by a battery, have been around since the 1960s, their prevalence is comparable to body-powered prostheses, as is their rejection rate [1]. Often-cited reasons for rejection include comfort, functionality, weight, and cost.

The newest generation of anthropomorphic myoelectric hands debuted roughly a decade ago and offers persons with amputation multiple grasp postures with the goal of enabling greater functionality and convenience while improving on aesthetics. This approach, however, imparts a greater economic cost on the user and can amount to a prosthetic device that is not utilized to its capacity or one that demands higher cognitive burden on the part of the user to fully access the widened feature set.

Leading neuroscientific theories tell us the brain organizes the control complexity of the hand motor system into synergies, or patterns of joint movement. This type of organization results in reduced dimensionality and was used as the basis for a robotic design concept called "Soft Syneriges" [2]. Employing underactuation and soft robotics techniques allows the robotic hand to follow the synergy trajectory until it encounters the surrounding environment and then mold to those environmental constraints. The Pisa/IIT SoftHand [3] was designed employing this concept and was shown to be a versatile robotic hand capable of grasping objects of a wide variety of shapes, sizes, and compliance. This work presents the steps towards translating this novel robotic hand into a prosthetic prototype and the first tests with subjects with amputations.

\section{Materials and Methods}

\subsection{Study Design}

The Pisa/IIT SoftHand was modified to render it more suitable for prosthetic use (dubbed the SoftHand Pro, see next section), and nine subjects with trans-radial amputations participated in an evaluation of the SoftHand Pro. All subjects signed an IRB-approved informed consent before taking part in the study. As part of a larger study that investigated subjects' performance and satisfaction with the SoftHand Pro, we measured hand function using the Jebsen Taylor test of hand 
function (Jebsen) [4]. Subjects completed the testing both before and after a brief (6-8 h) training period with the SoftHand Pro. For comparison, subjects also completed the same set of tests and surveys with their typical prosthesis prior to donning the SoftHand Pro.

\subsection{Study Device}

As mentioned above, the Pisa/IIT SoftHand was initially designed as a robotic hand intended for use on humanoid robots. The embodied intelligence of the SoftHand's mechanics suggested it had potential as a prosthetic hand that would not require complex control input from the user. In order to test this theory, it first had to be modified to be more suitable for prosthetic applications. In particular, the overall size of the hand was reduced to better approximate a large male hand. Further, the electronics were reduced in size, modified to interface with commercial electrodes (Otto Bock, Germany), and moved to the back of the hand to create a more compact design. To better interface with a prosthetic socket, a quick disconnect style wrist component was developed that allowed manual pronation and supination; further, the wrist was flexibly connected to the SoftHand Pro to allow passive wrist flexion and extension. Finally, a smaller and lower voltage motor was incorporated to allow use of a smaller, lighter-weight battery. For each subject, a custom prosthetic socket was built by a certified prosthetist.

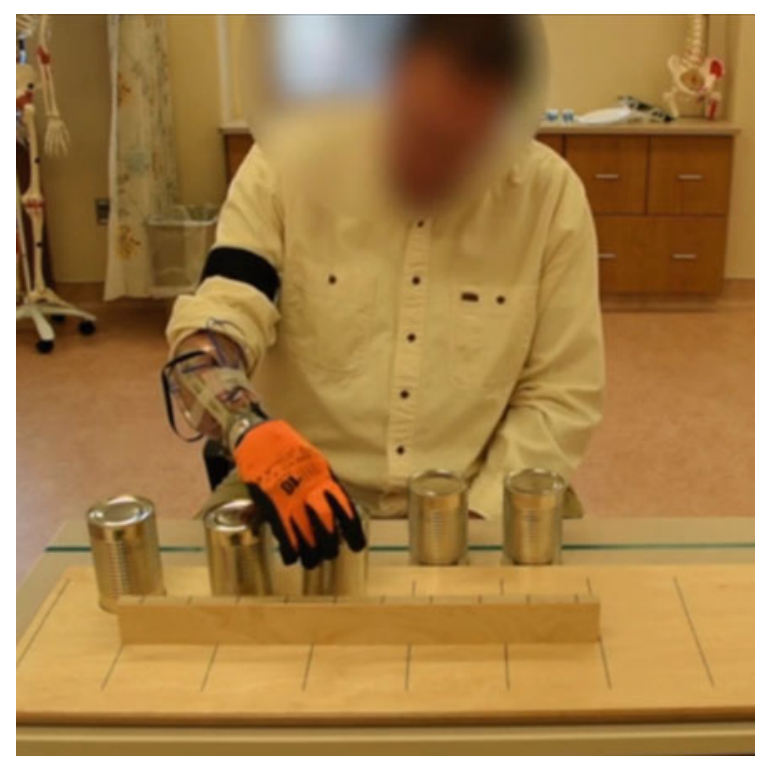

Fig. 1 A subject performing one of the Jebsen Taylor test of Hand Function subtasks 
Three myoelectric controllers were developed to enable the subject to control the SoftHand Pro even if he/she had limited control of the activity of his/her residual muscles. All three allowed proportional control of opening and closing of the hand and held position when the subject's muscle activity fell below a minimum threshold. The first mode (Differential) regulated prosthetic activity by balancing the input from the two signals, which allowed the user to fine-tune the signal and rapidly switch direction. To assist subjects with difficulty managing coactivation, the second mode (First Come, First Served, FCFS) responded to whichever muscle crossed the minimum threshold first; once it fell below threshold, direction could be switched. The final mode (FCFS +) was similar but required both signals to fall below the minimum threshold before the direction of activation could be changed; effectively, this requires the subject to relax both muscles before switching direction, thus enabling more precise but slower control (Fig. 1).

\section{Results}

Subjects' performance on the Jebsen following training was generally similar to performance with their typical prosthesis, see Fig. 2. Further, subjects showed marked improvement in their post-training scores compared to pre-training levels. Subjects were quickly able to learn to use the SoftHand Pro's deformability to aid in the grasping of irregular objects and to use environmental constraints to help shape the hand. However, the small object and checker stacking subtasks of the Jebsen still proved difficult and subjects underperformed in these tasks compared to their typical prosthesis.

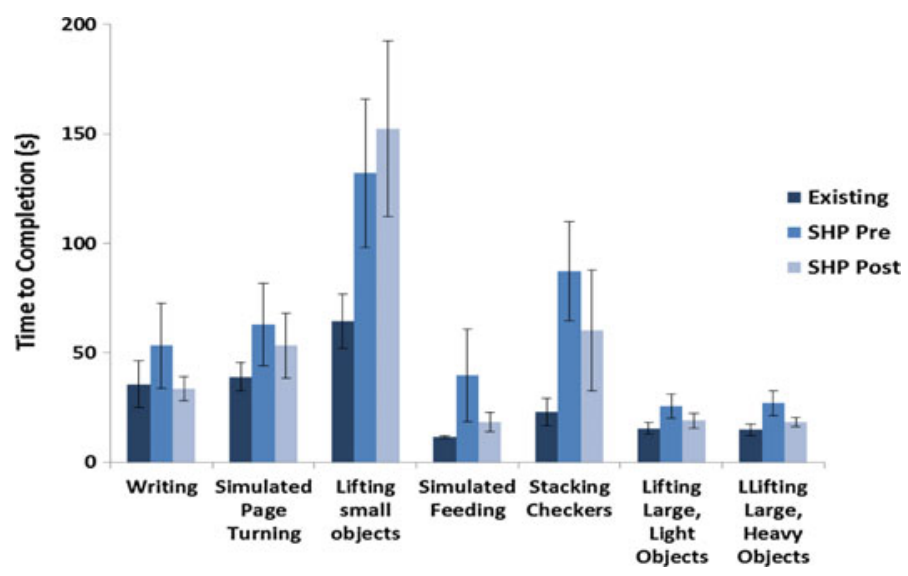

Fig. 2 Results from the Jebsen Taylor test of Hand Function. Note better performance is marked by shorter time to completion 


\section{Discussion and Conclusions}

Results from the initial translation of the Pisa/IIT SoftHand to the SoftHand Pro were quite positive and served to identify a key area for future work. While the SoftHand Pro was intuitive, highly functional, and easy to learn, subjects had greater difficulty with small object manipulation. In the future, this shortcoming will be addressed both in terms of targeted training and/or increased training duration as well as mechanical solutions that could aid small object grasping.

Acknowledgments Financial support was provided by the Grainger Foundation, National Institutes of Health NICHD grant R21HD081938-01, by the European Research Council under the Advanced Grant SoftHands "A Theory of Soft Synergies for a New Generation of Artificial Hands" no. ERC-291166, and by the European Union's Horizon 2020 Research and Innovation Programme under Grant Agreement "SoftPro," No. 688857.

\section{References}

1. E.A. Biddiss, T.T. Chau, Upper limb prosthesis use and abandonment: a survey of the last 25 years. Prosthet. Orthot. Int. 31(3), 236-257 (2007)

2. A. Bicchi, M. Gabiccini, M. Santello, Modeling natural and artificial hands with synergies. Philos. Trans. R. Soc. B 366, 3153-3161 (2011)

3. M.G. Catalano, G. Grioli, E. Farnioli, A. Serio, C. Piazza, A. Bicchi, Adaptive synergies for the design and control of the Pisa/IIT SoftHand. Int. J. Robot. Res. 33(5), 768-782 (2014)

4. R.H. Jebsen, N. Taylor, R.B. Trieschmann, M.J. Trotter, L.A. Howard, An objective and standardized test of hand function. Arch. Phys. Med. Rehabil. 50(6), 311-319 (1969) 